\title{
Histological Modifications Aging Aorta in Male Albino Rat
}

Abu-Dief EE ${ }^{1}$, Abdelrahim $\mathrm{EA}^{2^{*}}$ and Abdelrahim KM${ }^{2}$

${ }^{1}$ Department of Histology, Sohag Faculty of Medicine, Sohag University Egypt

${ }^{2}$ Department of Histology, Qena Faculty of Medicine, South Valley University, Egypt

*Corresponding author: Eman A. Abdelrahim, Professor of Histology, Sohag Faculty of Medicine, Sohag University Egypt, Tel: 20-97 3481101; E-mail: emaneweda@yahoo.com

Received date: February 15, 2016; Accepted date: April 15, 2016; Published date: April 25, 2016

Copyright: (C) 2016 Abu-Dief EE, et al. This is an open-access article distributed under the terms of the Creative Commons Attribution License, which permits unrestricted use, distribution, and reproduction in any medium, provided the original author and source are credited.

\begin{abstract}
Aging represents a major risk for vascular diseases. Diseases of the aorta causes serious back effects on the heart. This study aims to detect the histological changes of the aged aorta and to understand their underlying mechanisms to help in development of the prophylactic and treatment measures. Ten adult and ten aged male albino rats were used. Specimens from their thoracic aorta were prepared and stained with Hematoxylin and Eosin, Orcein for elastic laminae, and anti-actin antibody. Morphometric measurements for the thickness of the tunica intima and elastic laminae of the tunica media were performed. Aged aorta showed thickening of the tunica intima, thinning and fragmentation of elastic laminae, proliferation of medial smooth muscle with reduction of their actin myofibrils, and fibrosed tunica adventitia. Atherosclerosis was also detected with epithelioid changes in the medial smooth muscle and focal thickening of the intima. It is concluded that, aging is a risk factor for aortic atherosclerosis. Intimal thickening and proliferation of medial smooth muscle are the earliest signs. Thinning and fragmentation of elastic laminae, decreased contractile myofibrils of the aortic smooth muscle, and fibrosis of the tunica adventitia lead to aortic stiffness which interferes with its active function and shares in the age-related arterial remodeling.
\end{abstract}

Keywords: Aging aorta; Tunica intima; Elastic laminae; Vascular actin myofibrils; Atherosclerosis

\section{Introduction}

Aging represents a major risk factor for vascular disease development [1]. It is evident that aging results in well-defined phenotypic changes, which render the cardiovascular system prone to disease even in the absence of traditional risk factors such as hypertension, diabetes, and smoking. Moreover, if these risk factors are present, the age-related alterations in cellular homeostatic mechanisms render the aged vasculature more susceptible to their damaging effects. It is reported that cardiovascular diseases are the most common cause of death among the elderly patients in the Western world. Many factors can contribute to the occurrence of vascular aging such as endothelial dysfunction, vascular inflammation and arterial stiffness. Also, many underlying mechanisms may predispose to the age-related changes in the vasculature including oxidative stress, chronic low-grade inflammation and impaired endothelial progenitor cell function [2]. In addition, many theories can explain the aging-related changes in different organs and systems as the gene mutation theory, collagen deposition, wear and tear, and autoimmune theories [3]. Aorta which is the main and the largest artery of the body carries blood from the heart to all body organs and structures [4]. Histologically, the main thickness of the aortic wall is made by the tunica media. Its tunica media consists of concentrically arranged perforated elastic laminae, smooth muscle fibers, reticular fibers, proteoglycans and glycoprotein. Previous studies on the aged aorta revealed that it becomes thicker, stiffer, and less flexible with the advancement of age. This makes the blood pressure higher and makes the heart works harder with a consequent thickening (hypertrophy) of the cardiac muscle [3]. Clinically, these aging-related vascular changes may be manifested by angina, hypertension, transient ischemic attacks and strokes [5].
The current study was done to gain further insights into the histological changes of the aortic wall with aging, and discuss their underlying mechanisms. The study gives a particular concentration on the elastic laminae, and smooth muscle fibers of the tunica media. This study may help in better understanding of the vascular aging and in the development of the prophylactic and treatment strategies to avoid the susceptible associated serious clinical manifestations.

\section{Materials and Methods}

Twenty male albino rats were used in this study. They were purchased from the animal house of the Faculty of Medicine, Assiut University, Egypt. The animals were treated according to the international laws of the animal's rights. They were kept under normal day and night cycles at room temperature, fed rat chow ad libitum and allowed free access of water. They were divided equally into two groups according to their age. The adult group aged (3-4 months), and the aged group (1.5-2 years) according to Ohta and Ichimura [6]. The age of the animal was determined by the aid of the animal keeper. All animals used in this study were apparently healthy and of normal activity. The laboratory albino rat was selected in this study as it is the preeminent model for research into various areas, including cardiovascular disease, neurological disorders, neurobehavioral studies, cancer susceptibility and renal disease [7].

\section{Tissue preparation and staining techniques}

Animals were anaesthetized with ether and perfused by intracardiac infusion of saline until the flowing blood was cleared. The perfusion was completed with $10 \%$ neutral phosphate buffered formaldehyde. Specimens from the thoracic aorta of all animals were obtained, fixed in the same perfusate used, dehydrated, cleared and embedded in paraffin. Paraffin blocks were sectioned at 5-7 $\mu \mathrm{m}$ and stained with 
Page 2 of 6

Harris Hematoxylin and Eosin (H\&E) for routine histological examination. Orcein stain was done for demonstration of elastic laminae of the tunica media. Specimen preparation and staining techniques were done according to Drury and Wallington [8].

Some sections from all specimens (both adult and aged) were picked upon positive slides for the immuno-histochemical study which was done using the anti-actin antibody; actin, smooth muscle Ab-1 (Clone 1A4) Mouse Monoclonal Antibody (Thermo Fisher Scientific, Fremont, CA, USA). Ab-1 stains smooth muscle in vessel wall, gut wall and myometrium. After deparaffinization of the sections, antigen retrieval was done by boiling tissue sections in 1mM EDTA, $\mathrm{pH} 8.0$ for 10-20 minutes followed by cooling at room temperature for 20 minutes. Sections were then pretreated with hydrogen peroxide block for 15 minutes to reduce non-specific background staining due to endogenous peroxidase, washed by phosphate buffer, followed by incubation in the ultra $\mathrm{V}$ block for 5 minutes at room temperature to block the non-specific background staining. The primary antibody (anti-actin antibody; actin, smooth muscle Ab-1) was diluted at 1:200 in antibody diluent. Sections were incubated in the primary antibody for 30 minutes at room temperature. The positive control was the aorta of the normal adult animals. The reaction was visualized using; UltraVision ONE Detection System, HRP Polymer \& DAB Plus Chromogen (Thermo Fisher Scientific, Fremont, CA, USA). The procedure was done according to the manufacture instructions. After completion of the reaction, counter-staining was done using Hematoxylin. The changes in the immunohistochemical reaction of the smooth muscle fibers of the aortic wall of the aged animals were detected through a comparative examination of the corresponding tissues of the adult animals.

\section{Morphometric study}

Morphometric study was done by measuring the thickness of both the tunica intima and the elastic laminae of the tunica media in the wall of the aorta in both adult and aged animals. Measuring was done from, at least, three $\mathrm{H} \& \mathrm{E}$ stained slides for each animal, both adult and aged. For each slide, measuring of the thickness of the tunica intima was done from 3-5 points along the circumference of the aortic section. The measured area was the area that extends from the endothelial surface to the first detected elastic lamina. The average value for the tunica intima was lastly calculated for each slide. Finally, the thickness of the tunica intima for each animal was estimated by calculating the average value of the three measured slides. In our study, we excluded the pathological slides i.e. those with atheromatous plaques that showed marked intimal thickening.

Regarding the measuring of the thickness of the elastic laminae, it was done by measuring all the elastic laminae from three Orcein stained slides for each animal. The average value for each slide was calculated and the average value of the three slides for each animal was estimated to be used in the statistical study. All measures were done only from the exact transverse aortic sections excluding the oblique sections. Measures were made using Leica EZ4D stereomicroscope (Leica DM 1000) with internal digital camera using $\times 40$ objective lens. The microscope is equipped with Leica EC3 digital camera, which is programmed with LAS EZ (Leica Application Suite Educational Zoom) software image analyzer.

\section{Statistical analysis}

Statistical analysis of data was done using Microsoft Excel 2013 through the descriptive statistics (summary statistics) and analysis of variance (ANOVA) single factor test. P-value $<0.05$ was considered statistically significant and $\mathrm{p}$-value $<0.001$ was considered statistically highly significant.

\section{Results}

\section{Hematoxylin and Eosin}

Examination of the aorta of adult male albino rats revealed a thin tunica intima with a hardly detected subendothelial connective tissue making the tunica intima hardly distinguished from the tunica media. The endothelial lining of the tunica intima was very thin, and was formed of extremely thin flattened endothelial cells with flattened nuclei.

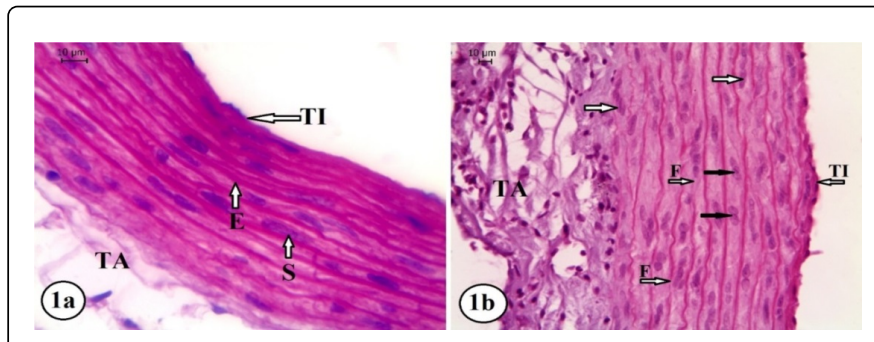

Figure 1: (a) A photomicrograph of the aorta of adult male albino rat shows a thin smooth tunica intima (TI), regularly arranged parallel elastic laminae (E) of the tunica media with regularly arranged spindle smooth muscle fibers (S) in between, and tunica adventitia (TA) which is formed of loose connective tissue. (H\&E, $\times 400$ ). (b) A photomicrograph of the aorta of aged male albino rat shows a relative diffuse thickening of the tunica intima (TI) with a proliferating endothelium and a relatively rough surface, proliferating smooth muscle fibers of the tunica media where some are binucleated (black arrows) and others are multinucleated (white arrows), thin fragmented (F) elastic laminae, and fibrosed tunica adventitia (TA). (H\&E, ×200).

The surface of the tunica intima that lines the lumen of the aorta was very smooth. The elastic laminae of the tunica media were regularly arranged and parallel. The narrow spaces between them contained an acidophilic matrix and regularly arranged spindle smooth muscle fibers with oval to flattened nuclei. The tunica adventitia was formed of loose connective tissue in Figure 1a. In comparison, the aorta of aged male albino rats revealed a relative diffuse thickening of the tunica intima to the extent that it could be easily distinguished from the tunica media. The luminal surface was relatively rough and the endothelial cells were proliferating as their nuclei were close to each other. The elastic laminae of the tunica media were thin with a relatively wide spaces between them. Some of them were fragmented. The smooth muscle fibers in between the elastic laminae were proliferating. Most of them were binucleated while others were multinucleated. The tunica adventitia was thick and fibrosed Figure 1b. Occasionally, histopathological changes were detected in the aorta of aged rats in the form of atheromatous plaques. They were usually detected in the tunica media causing destruction the elastic laminae (Figure 2a and 2c). The overlying tunica intima showed 
a marked focal thickening with an obviously rough luminal surface (Figure 2a and 2b). It also showed invasion of the subendothelial connective tissue by proliferating endothelial cells that appeared as groups of flattened cells just beneath the intimal surface (Figure $2 b$ ). Concomitant with these atheromatous plaques, the subendothelial connective tissue was also invaded by groups of large rounded epithelioid cells with a basophilic cytoplasm, a rounded vesicular eccentric nucleus and prominent juxta-nuclear Golgi image (Figure 2b). Epithelioid cells of the same criteria were detected in the tunica media in between the elastic laminae just around the atheromas, together with undifferentiated large irregular basophilic cells with large irregular nuclei (Figure $2 \mathrm{c}$ and $2 \mathrm{~d}$ ). The matrix between elastic laminae particularly around the atheromas was basophilic (Figure 2b-2d).

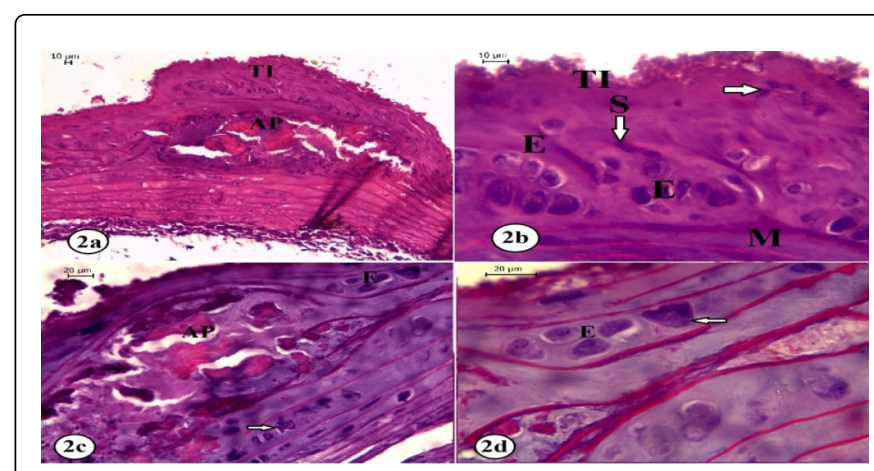

Figure 2: Photomicrographs of the aorta of aged male albino. (a) Shows a large atheromatous plaque (AP) destructing the upper layers of the tunica media with a marked focal thickening of the tunica intima (TI). Notice that the endothelial lining lacks its smooth surface. (H\&E, $\times 100)$. (b) A magnified part shows destruction of the upper parts of the elastic laminae and invasion of the subendothelial connective tissue by groups of large rounded epithelioid cells (E) with a basophilic cytoplasm, a rounded vesicular eccentric nucleus, and a prominent juxta-nuclear Golgi image. Notice that there are proliferating endothelial cells (arrow) and proliferating smooth muscle fibers (S) underneath the destructed elastic laminae. Notice that tunica intima (TI) lacks its smooth endothelial lining and that the matrix $(\mathrm{M})$ between elastic laminae is basophilic. $(\mathrm{H} \& \mathrm{E}, \times 200)$. (c) Shows a large atheromatous plaque (AP) destructing the tunica media, the spindle shaped smooth muscle fibers around the atheroma are lacking with the presence of round to oval epithelioid cells (E) instead, some of them appear irregular (arrow). (H\&E, $\times 200$ ). (d) A magnified part shows a group of epithelioid cells (E) in the matrix between the elastic laminae. The matrix appears basophilic, the elastic laminae are thin, and the epithelioid cells are rounded with basophilic cytoplasm and eccentric vesicular nucleus. Notice the presence of an undifferentiated large irregular basophilic cell with large irregular eccentric nucleus (arrow) among the epithelioid cells. (H\&E, $\times 400)$.

\section{Immunohistochemical stain}

The immune staining was done using the anti-actin antibody. Aorta of adult male albino rats showed a highly actin immune-positive smooth muscle fibers of the tunica media that looked packed with the immune-positive actin myofibrils Figure $3 \mathrm{a}$ and $3 \mathrm{~b}$. Aorta of aged male albino rat showed an obvious reduction in the actin immune-positivity of the smooth muscle fibers of the tunica media, appeared by marked reduction of the brown color of the immune-positive myofibrils Figure $3 \mathrm{c}$. The immune-stained actin myofibrils were only confined to localized areas of the smooth muscle fibers Figure $3 \mathrm{~d}$.

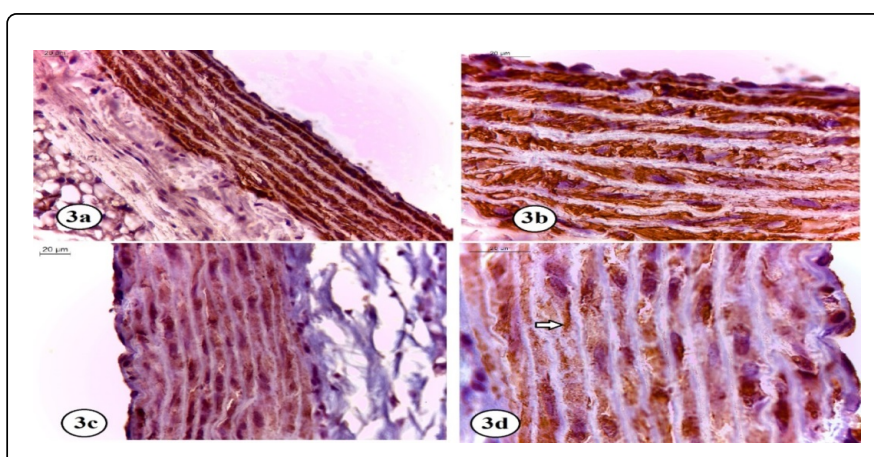

Figure 3: (a) A photomicrograph of the aorta of adult male albino rat shows a highly actin immune-positive smooth muscle fibers of the tunica media. (Immune staining with anti-actin antibody, $\times 200$ ). (b) A magnified part shows that the smooth muscle fibers are packed with the immune-stained actin myofibrils. (Immune staining with anti-actin antibody, $\times 400$ ). (c) A photomicrograph of the aorta of aged male albino rat shows an obvious reduction in the actin immune-positivity of the smooth muscle fibers of the tunica media. (Immune staining with anti-actin antibody, $\times 200$ ). (d) A magnified part shows that the immune-stained actin myofibrils are confined to localized area of the smooth muscle fibers (arrow). (Immune staining with anti-actin antibody, $\times 400$ ).

Immune-staining of the aged atheromatous aortas revealed that the epithelioid cells of the tunica intima Figure 4a and tunica media Figure $4 \mathrm{~b}$, as well as the undifferentiated cells Figure $4 \mathrm{~b}$, all were actin immune-positive (Figure 4).

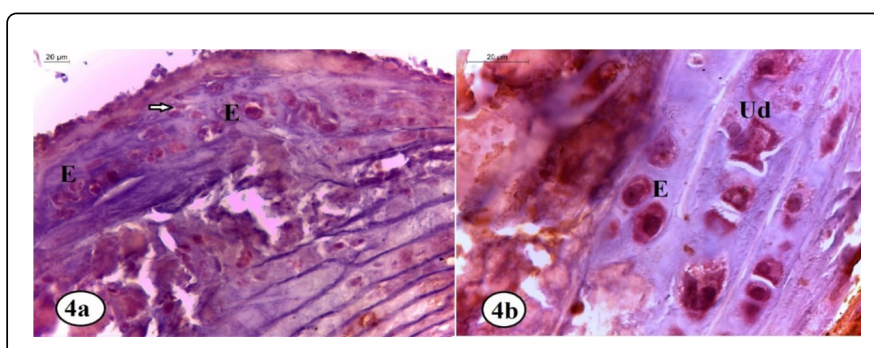

Figure 4: Photomicrographs of the aorta of aged male albino rat. (a) Shows immune-positive epithelioid cells (E) in the subendothelial connective tissue of the tunica intima and immune-positive proliferating endothelial cells (arrow). (Immune staining with antiactin antibody, $\times 200$ ). (b) Shows immune-positive epithelioid cells (E) and undifferentiated (Ud) of the tunica media. (Immune staining with anti-actin antibody, $\times 1000)$.

\section{Orcein stain}

This stain was done to detect the aging-related changes in the elastic laminae of the tunica media. Elastic laminae of adult rats were thick parallel regularly arranged Figure $5 \mathrm{a}$. In comparison, those of old rats were relatively thin with areas of fragmentation and in some areas they formed a network of thin fibrils Figure $5 b$. 
Page 4 of 6
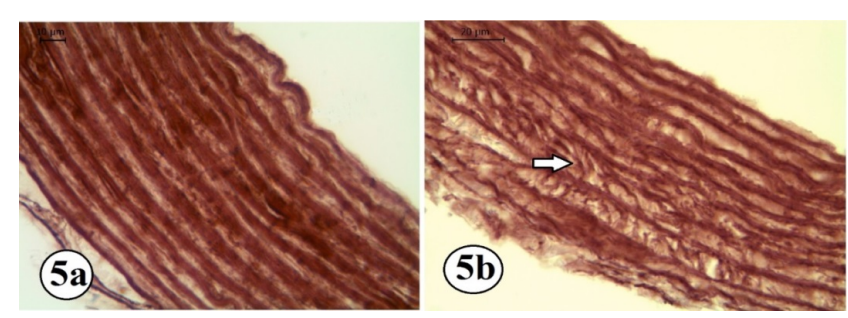

Figure 5: (a) A photomicrograph of the aorta of adult male albino rat shows thick parallel regularly arranged elastic laminae. (Orcein, $\times 400$ ). (b) A photomicrograph of the aorta of aged male albino rat shows relatively thin elastic laminae that appear fragmented and form a network of thin fibrils in some areas (arrow). (Orcein, $\times 400)$.

\section{Morphometric Results}

Measuring of the thickness of the tunica intima revealed that in adult animals the thickness was ranged from 1.248 to 3.353 with a mean value of $2.331 \pm$ standard deviation (SD) 0.779. In aged animals the thickness was ranged from 3.766 to 6.238 with a mean value of $5.0175 \pm$ standard deviation $(\mathrm{SD})$ 0.741. $\mathrm{P}$ value was $1.6 \mathrm{E}-06$ $(0.0000016)$ which is statistically highly significant. The results are summarized in Table 1 and presented in Figure 6 shows histogram 1.

\begin{tabular}{|l|l|l|l|}
\hline Animal & Range & Mean \pm SD & P value \\
\hline Adult & $1.248-3.353$ & $2.331 \pm 0.779$ & \multirow{2}{*}{0.0000016} \\
\cline { 1 - 3 } Aged & $3.766-6.238$ & $5.0175 \pm 0.741$ & \\
\hline
\end{tabular}

Table 1: Summarizes values of the thickness of tunica intima in both adult and aged.

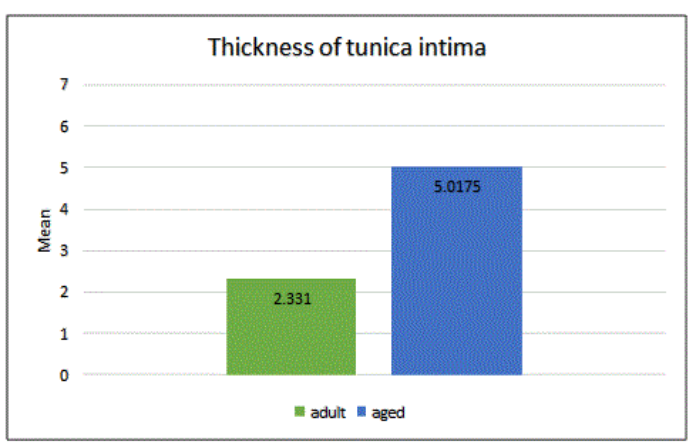

Figure 6: Histogram 1 shows a bar chart presentation of the mean value of the thickness of the tunica intima in both adult and aged \pm SD.

Measuring of the thickness of the elastic laminae revealed that in adult animals the thickness was ranged from 4.18 to 6.57 with a mean value of $5.366 \pm$ standard deviation (SD) 0.87 . In aged animals the thickness was ranged from 2.494 to 4.366 with a mean value of $3.32 \pm$ standard deviation (SD) 0.634. P value was 6.79 E-07 (0.000000679) which is statistically highly significant. The results are summarized in Table 2 and presented in Figure 7, histogram 2.

\begin{tabular}{|l|l|l|l|}
\hline Animal & Range & Mean \pm SD & P value \\
\hline Adult & $4.18-6.57$ & $5.366 \pm 0.87$ & \multirow{2}{*}{0.000000679} \\
\cline { 1 - 3 } Aged & $2.494-4.366$ & $3.32 \pm 0.634$ & \\
\hline
\end{tabular}

Table 2: Summarizes values of the thickness of elastic laminae in both adult and aged.

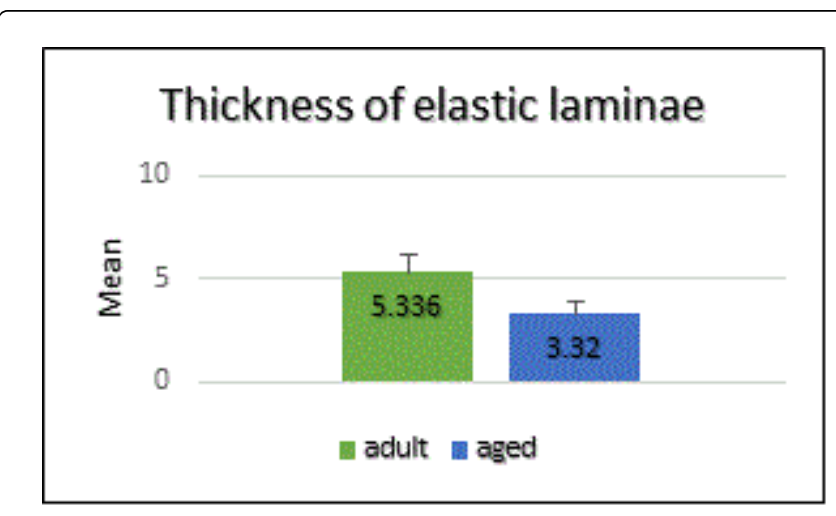

Figure 7: Histogram 2: Shows a bar chart presentation of the mean value of the thickness of the elastic laminae in both adult and aged \pm SD.

\section{Discussion Occasionally, Histopathological Change}

The first noticeable difference between aged and adult aorta starting from inside outward was the obvious statistically highly significant intimal thickening concomitant with proliferation of the endothelial cells. In fact, measuring the intimal thickness in young animals was very hard. In this aspect it is important to mention that some authors thought that arterial intima in some animal species particularly small animals does not form unless the vessel first undergoes trauma [9].

Similar to the observed increase in intimal thickness in aged rat aorta, diffuse intimal hypertrophy, in old age humans, was seen by Gupta et al. [4]. Moreover, intimal thickening was observed in large arteries of human aged donors with increased cell number in both intimal thickening and tunica media [10].

Intimal thickening is referred to as neointima. Neointima is an intima that forms in response to injury. Neointimal formation occurs in all arteries as a response to wide variety of injuries, however, neointimal formation is a characteristic of responses to injury in the smooth muscle-rich vessels. Arterial injury may also lead to smooth muscle cell migration and proliferation in the intima [11]. Once smooth muscle cells arrive in the intima of the artery, neointimal cells closest to the lumen may replicate for weeks to months [12].

Intimal smooth muscle cells might belong to a unique lineage either derived from a common precursor or differentiated from typical smooth muscle cells. Theories of both atherosclerosis and restenosis simplistically assume that an increase in mass of the intima is the cause of loss of lumen caliber [11].

Another very important finding in the intima of aged aorta was the lacking of the smooth endothelial surface. It might be due to fat accumulation and calcification of the intima that occurs throughout life [11] 
It seems that intima is a soil for arterial pathology mainly for atherosclerosis as considered by Schwartz et al. [11] and that intimal thickening is one of the earliest signs of atherosclerosis. Gupta et al. [4] reported that intimal thickening is the most important aging change for it appears to carry the seeds of arterial disease. The elements added to the intima in time include fibrocytes, smooth muscle cells collagen fibers and ground substance rich in mucopolysacchrides. Molecular mechanisms underlying the progress of arterial pathology following intimal thickening need a good understanding to develop the preventive and treatment strategies at the proper time.

One the most striking observations in this study was the proliferation of the smooth muscle fibers of the tunica media which is a criterion of smooth muscle stem cells.

Smooth muscle cells are the main component of the arterial wall carrying out several physiological actions such as blood pressure control and blood volume distribution by contraction and relaxation of the vessels [12]. Changes of the biological properties of vascular smooth muscle cells were observed in large arteries of human aged donors. In these arteries, accumulation of smooth muscle cells with a stem phenotype was observed. Stem marker expression was found to characterize smooth muscle cells during developmental growth and atherosclerosis. Therefore, accumulation of smooth muscle cells with a stem phenotype in old age contributes to the age-dependent adverse arterial remodeling [1].

Age-dependent increase in vascular smooth muscle cell proliferation is thought to contribute to the pathology of atherosclerotic diseases. Mitogen-activated protein kinases play a possible role in vascular smooth muscle cell proliferation and neointimal formation in the context of aging. Age-related increase in the level of these proteins was associated with a higher cellular proliferative index and a significant increase in neointimal formation in old animals. Inhibition of these protein kinases could represent a novel therapeutic avenue against atherosclerotic diseases [13].

In the current study, atheromatous plaques were detected in the tunica media of some sections of aged animals concomitant with overlying marked focal intimal thickening. The plaques were also associated with proliferative changes of both endothelial cells and smooth muscle cells. Moreover, the surrounding smooth muscle cells were transformed into epithelioid cells in addition to the appearance of irregular shaped undifferentiated cells which were immune positive to anti-actin antibody. Also, the matrix in between the thin and destroyed elastic laminae in these sections appeared highly basophilic.

It was previously reported that aging has been indicated as one of the major risk factors for the development of atherosclerotic lesions [14]. In this aspect, smooth muscle cells, in particular those of the modulated i.e. synthetic phenotype which are able to proliferate and synthesize matrix proteins, are considered to play an important role in lesion progression [13]. This might also explain the increased basophilia of the matrix in between elastic laminae observed with atheroma in this study that might result from the increased secretion of matrix mucopolysaccharides by the smooth muscle cells.

Immune-staining of smooth muscle fibers of adult aorta using the anti-actin antibody showed that muscle fibers were packed with actin, while those of aged aorta showed marked reduction of actin myofibrils. Also aged aorta with atheroma showed actin positive flattened and epithelioid cells in the thickened tunica intima.
Previous study of large arteries of human aged donors showed increased cell number in the intimal thickening and tunica media and that intimal cells co-expressed $\alpha$-smooth muscle actin. Also, in vitro, old rat aortic smooth muscle cells proliferated and migrated more with less $\alpha$-smooth muscle actin and myosin compared to young ones [1]. It was obvious that concomitant with smooth muscle proliferation there was a reduction of their content of actin myofibrils. This means that these muscles are ill-differentiated and have a less contractile power which certainly has bad effects on the performance and compliance of the artery. Therefore, the aged vascular smooth muscles have an increased proliferative and secretory activities on the expense of the contractile activity.

In the present study, an obvious statistically highly significant thinning of elastic laminae was observed in aged aortas. In addition there were areas of fragmentation of these elastic laminae associated with the formation of networks of fine fibrils in between them.

In agreement with our finding Gupta et al. [4] observed that in old age, arterial elastic laminae became thinned and showed fragmentation with occasional clumping. Fritze et al. [15] also previously detected that aging was accompanied by a destruction of the elastic vascular structure as observed by laser scanning microscopy and confirmed by histology. However, analysis of tropoelastin expression suggested that elastogenesis occurs throughout life with constantly decreasing levels.

In the current study, networks of thin fibrils were observed in between elastic laminae. Greenberg [16] mentioned that with age a cross linking microfibrillar type of elastin increases on the expense of the thick regularly arranged parallel elastic laminae. Moreover, Francis et al. [18] noticed that degenerative changes appeared in elastic fibers in old age and they were associated with a reparative process that was indicated by the development of thinner fibers irregularly oriented around the original membrane $[19,20]$.

Lastly, tunica adventitia of aged aorta was obviously fibrosed that might share in the age-related arterial stiffness [2] and remodeling [1].

\section{Conclusion}

It is concluded that,

- Aging is a major risk factor for the development of atherosclerosis.

- The aged aorta showed intimal thickening and proliferation of medial smooth muscles with reduction of their actin myofibrils.

- These changes are considered the earliest and may be the controllable signs of atherosclerosis.

- Moreover, aging is associated with thinning and fragmentation of elastic laminae and fibrosis of the tunica adventitia that leads to stiffness and hardening of the artery.

- All these changes certainly could interfere with the active function of the aorta and are parts of the age-related arterial remodeling that may have serious drawbacks on the cardiac muscle.

\section{References}

1. Ferlosio A, Arcuri G, Doldo E, Scioli MG, De Falco S, et al. (2012) Agerelated increase of stem marker expression influences vascular smooth muscle cell properties, Atherosclerosis 224: 51-57.

2. Ungvari Z, Kaley G, de Cabo R, Sonntag WE, A Csiszar (2010) Mechanisms of Vascular Aging: New Perspectives (Review Article), J Gerontol A Biol Sci Med Sci 65: 1028-1041. 
Citation: Abu-Dief EE, Abdelrahim EA, Abdelrahim KM (2016) Histological Modifications Aging Aorta in Male Albino Rat. J Cytol Histol 7: 408.

Page 6 of 6

3. Gupta SD, Gupta SK, Pal DK, Sarawagi R, Gupta P (2011) Microscopic study of aorta in relation of different age groups: an observational study, Int J Biol Med Res 2: 398-403.

4. Waugh A, Grant A (2006) Ross and Wilson Anatomy and Physiology in Health and Illness. (10thedn), Churchill Livingstone. Section 2, 5: 76-129.

5. Minaker KL (2007) Common clinical sequelae of aging, In Goldman L, Ausielo D (eds.) Cecil Medicine. (23rdedn) Philadelphia, Pa: Saunders Elsevier. Ch. 23.

6. Mescher AL (2010) Junqueira's Basic Histology. Textbook and Atlas. (12thedn), Mc Graw-Hill. Ch. II, pp: 185-202.

7. Ohta Y, Ichimura K (2000) Changes in epidermal growth factor receptors in olfactory epithelium associated with aging, Ann Otol Rhinol Laryngol 109: 95-98.

8. Sengupta P (2011) A Scientific Review of Age Determination for a Laboratory Rat: How Old is it in Comparison with Human Age? Review Biomedicine International 2: 81-89

9. Drury RAB, Wallington EA (1980) Carleton's histology technique, (5thedn) Oxford University Press, Oxford-New York-Toronto. pp: 241-242.

10. French JE (1966) Atherosclerosis in relation to the structure and function of the arterial intima, with special reference to the endothelium. Int Rev Exp Pathol 5: 253-353.

11. Schwartz SM, deBlois D (1995) The Intima: A New Soil

12. Clowes AW, Clowes MM, Reidy MA (1986) Kinetics of cellular proliferation after arterial injury, III: endothelial and smooth muscle growth in chronically denuded vessels. Lab Invest 54: 295-303.
13. Stoehr R, Federici M (2012) Do stem cells cause aging-related intimal medial thickening? Atherosclerosis 244: 39-40.

14. Gennaro G, Ménard C, Giasson E, Michaud SE, Palasis M, et al. (2003) Role of p44/p42 MAP kinase in the age-dependent increase in vascular smooth muscle cell proliferation and neointimal formation, Arterioscler. Thromb Vasc Biol 23: 204-210.

15. Spagnoli LG, Orlandi A, Mauriello A, De Angelis C, Ramacci MTI (1992) Age-dependent increase of rabbit aortic atherosclerosis. A morphometric approach. Pathol Res Pract 188: 637-642.

16. Fritze O, Romero B, Schleicher M, Jacob MP, Oh DY, et al. (2012) Agerelated changes in the elastic tissue of the human aorta. J Vasc Res 49: 77-86.

17. Greenberg SR (1986) The association of medial collagenous tissue with atheroma formation in the aging human aorta as revealed by a special technique. Histol Histopathol 4: 323-326.

18. Francis G, John R, Thomas J (1973) Biosynthetic pathway of desmosines in elastin. Biochem J 136: 45-55.

19. Schwartz SM, deBlois D, O'Brien ER (1995)"Soil for Atherosclerosis and Restenosis", Circulation Research 77: 445-465.

20. Schwartz RS, Edwards WD, Huber KC, Antoniades LC, Bailey KR, et al. (1993) Coronary restenosis: prospects for solution and new perspectives from a porcine model. Mayo Clin Proc 68: 54-62. 